\title{
Stability Analysis of Stochastic Reaction-Diffusion Cohen-Grossberg Neural Networks with Time-Varying Delays
}

\author{
Chuangxia Huang, ${ }^{1}$ Xinsong Yang, ${ }^{2}$ and Yigang $\mathrm{He}^{3}$ \\ ${ }^{1}$ College of Mathematics and Computing Science, Changsha University of Science and Technology, \\ Changsha, Hunan 410076, China \\ ${ }^{2}$ Department of Mathematics, Honghe University, Mengzi, Yunnan 661100, China \\ ${ }^{3}$ College of Electrical and Information Engineering, Hunan University, Changsha, Hunan 410082, China \\ Correspondence should be addressed to Chuangxia Huang, cxiahuang@126.com
}

Received 28 July 2009; Accepted 16 September 2009

Recommended by Yong Zhou

This paper is concerned with $p$ th moment exponential stability of stochastic reaction-diffusion Cohen-Grossberg neural networks with time-varying delays. With the help of Lyapunov method, stochastic analysis, and inequality techniques, a set of new suffcient conditions on $p$ th moment exponential stability for the considered system is presented. The proposed results generalized and improved some earlier publications.

Copyright (c) 2009 Chuangxia Huang et al. This is an open access article distributed under the Creative Commons Attribution License, which permits unrestricted use, distribution, and reproduction in any medium, provided the original work is properly cited.

\section{Introduction}

Since the seminal work for Cohen-Grossberg neural networks by Cohen and Grossberg [1], theoretical understanding of neural network dynamics has advanced greatly. The model can be described by a system of ordinary differential equations

$$
\dot{x}_{i}(t)=-\alpha_{i}\left(x_{i}(t)\right)\left[\beta_{i}\left(x_{i}(t)\right)-\sum_{j=1}^{n} a_{i j} g_{j}\left(x_{j}(t)\right)\right],
$$

where $t \geq 0, n \geq 2 ; n$ corresponds to the number of units in a neural network; $x_{i}(t)$ denotes the potential (or voltage) of cell $i$ at time $t ; f_{j}(\cdot)$ denotes a nonlinear output function between cell $i$ and $j ; \alpha_{i}(\cdot)>0$ represents an amplification function; $\beta_{i}(\cdot)$ represents an appropriately behaved function; the $n \times n$ connection matrix $A=\left(a_{i j}\right)_{n \times n}$ denotes the strengths of connectivity 
between cells, and if the output from neuron $j$ excites (resp., inhibits) neuron $i$, then $a_{i j} \geq 0$ (resp., $a_{i j} \leq 0$ ).

During hardware implementation, time delays do exist due to finite switching speed of the amplifiers and communication time and, thus, delays should be incorporated into the model equations of the network. For model (1.1), Ye et al. [2] introduced delays by considering the following delay differential equations:

$$
\dot{x}_{i}(t)=-\alpha_{i}\left(x_{i}(t)\right)\left[\beta_{i}\left(x_{i}(t)\right)-\sum_{j=1}^{n} a_{i j} f_{j}\left(x_{j}\left(t-\tau_{j}\right)\right)\right] .
$$

Some other more detailed justifications for introducing delays into model equations of neural networks can be found in [3-13] and references therein. It is seen that (1.2) is quite general and it includes several well-known neural networks models as its special cases such as Hopfield neural networks, cellular neural networks, and bidirectional association memory neural networks (see, e.g., [14-18]).

In addition to the delay effects, stochastic effects constitute another source of disturbances or uncertainties in real systems [19]. A lot of dynamical systems have variable structures subject to stochastic abrupt changes, which may result from abrupt phenomena such as stochastic failures and repairs of the components, changes in the interconnections of subsystems, and sudden environment changes. In the recent years, the stability investigation of stochastic Neural Networks is interesting to many investigators, and a large number of stability criteria of these systems have been reported [20-30]. The stochastic model can be described by a system of stochastic differential equations

$$
\begin{aligned}
\mathrm{d} x_{i}(t)= & -\alpha_{i}\left(x_{i}(t)\right)\left[\beta_{i}\left(x_{i}(t)\right)-\sum_{j=1}^{n} a_{i j} g_{j}\left(x_{j}(t)\right)-\sum_{j=1}^{n} b_{i j} g_{j}\left(x_{j}\left(t-\tau_{j}(t)\right)\right)\right] \mathrm{d} t \\
& +\sum_{j=1}^{n} \sigma_{i j}\left(t, x_{i}(t), x_{j}\left(t-\tau_{j}(t)\right)\right) \mathrm{d} \omega_{j}(t) .
\end{aligned}
$$

However, besides delay and stochastic effects, diffusion effect cannot be avoided in the neural networks when electrons are moving in asymmetric electromagnetic fields [31], so we must consider the activations vary in space as well as in time. In [32-36], authors have considered the stability of reaction-diffusion neural networks with constant or time-varying delays, which are expressed by partial differential equations. To the best of our knowledge, few authors have considered the problem of $p$ th moment stability for stochastic Cohen-Grossberg neural networks with both time-varying delays and reactiondiffusion terms. Motivated by the above discussions, in this paper, we consider the stochastic reaction-diffusion Cohen-Grossberg neural networks with time-varying delays described by 
the following stochastic partial differential equations:

$$
\begin{aligned}
\mathrm{d} y_{i}(x, t)= & \sum_{k=1}^{m} \frac{\partial}{\partial x_{k}}\left(D_{i k} \frac{\partial y_{i}}{\partial x_{k}}\right) \mathrm{d} t-\alpha_{i}\left(y_{i}(x, t)\right) \\
& \times\left[\beta_{i}\left(y_{i}(x, t)\right)-\sum_{j=1}^{n} a_{i j}(t) g_{j}\left(y_{j}(x, t)\right)-\sum_{j=1}^{n} b_{i j}(t) g_{j}\left(y_{j}\left(x, t-\tau_{j}(t)\right)\right)\right] \mathrm{d} t \\
& +\sum_{j=1}^{n} \sigma_{i j}\left(t, y_{i}(x, t), y_{j}\left(x, t-\tau_{j}(t)\right)\right) \mathrm{d} \omega_{j}(t)
\end{aligned}
$$

where

$$
\begin{gathered}
\frac{\partial y_{i}}{\partial n}:=\left(\frac{\partial y_{i}}{\partial x_{1}}, \ldots, \frac{\partial y_{i}}{\partial x_{m}}\right)^{T}=0, \quad t \geq 0, i=1, \ldots, n, x \in \Omega, \\
y_{i}=y_{i}(x, s)=\phi_{i}(x, s), \quad-\infty<s \leq 0, x \in \Omega .
\end{gathered}
$$

In the above model, $n \geq 2$ corresponds to the number of units in the neural network, $x=$ $\left(x_{1}, \ldots, x_{m}\right)^{T}$ is space variable, and $y_{i}(x, t)$ denotes the state variable of cell $i$ at time $t$ in space variable $x$; smooth function $D_{i k}=D_{i k}(x, y, t) \geq 0$ is a diffusion operator; $\Omega$ is a compact set with smooth boundary $\partial \Omega$ and the measure mes $\Omega>0$ in $R^{m}$,

$$
\left.\frac{\partial y_{i}}{\partial n}\right|_{\partial \Omega}=0
$$

and $\phi_{i}(x, s)$ are the boundary value and initial value, respectively; $a_{i j}(t)$ and $b_{i j}(t)$ denote the strengths of connectivity between cell $i$ and $j$ at time $t$, respectively; $\tau_{j}(t)$ is time delay and satisfies $0 \leq \tau_{j}(t) \leq \tau ; \sigma=\left(\sigma_{i j}\left(t, y_{i}(x, t), y_{j}\left(x, t-\tau_{j}(t)\right)\right)\right)_{n \times n}$ is the diffusion coefficient matrix, and $\omega(t)=\left(\omega_{1}(t), \ldots, \omega_{n}(t)\right)^{T}$ is an $n$-dimensional Brownian motion defined on a complete probability space $(\boldsymbol{\Omega}, \boldsymbol{F}, \mathbf{P})$ with a natural filtration $\left\{\mathcal{F}_{t}\right\}_{t \geq 0}$ by standard Brownian motion $\{w(s): 0 \leq s \leq t\}$. As a standing hypothesis, we assume that $g_{j}(\cdot)$ and $\sigma(t, \cdot, \cdot)$ satisfy the Lipschitz condition and the linear growth condition and that (1.4) has a solution on $t \geq 0$ for the initial conditions.

The remainder of this paper is organized as follows. In Section 2, the basic notations and assumptions are introduced. In Section 3, criteria are proposed to determine $p$ th moment exponential stability for the stochastic Cohen-Grossberg neural networks with timevarying delays and reaction-diffusion term. An illustrative example is given to illustrate the effectiveness of the obtained results in Section 4. We also conclude this paper in Section 5. 


\section{Preliminaries}

For any $y(x, t)=\left(y_{1}(x, t), \ldots, y_{n}(x, t)\right)^{T} \in R^{n}$, we define

$$
\begin{gathered}
\left\|y_{i}(x, t)\right\|_{p}=\left[\int_{\Omega}\left|y_{i}(x, t)\right|^{p} \mathrm{~d} x\right]^{1 / p}, \\
\|y(x, t)\|=\left[\sum_{j=1}^{n}\left\|y_{i}(x, t)\right\|_{p}^{p}\right]^{1 / p} .
\end{gathered}
$$

As usual, we will also assume that the following conditions are satisfied.

$\left(H_{1}\right)$ There exist positive constants $\underline{\alpha}_{i}, \bar{\alpha}_{i}$, such that

$$
\underline{\alpha}_{i} \leq a_{i}\left(y_{i}(x, t)\right) \leq \bar{\alpha}_{i}
$$

$\left(H_{2}\right)$ For each $i \in\{1, \ldots, n\}$, there exists positive constant $G_{i}$, such that

$$
\left|g_{i}(u)-g_{i}(v)\right| \leq G_{i}|u-v|, \quad \forall u, v, \in R .
$$

$\left(H_{3}\right)$ There exist positive functions $\gamma_{j}(t)$, such that

$$
y_{j}(x, t) \beta_{j}\left(y_{j}(x, t)\right) \geq \gamma_{j}(t) y_{j}^{2}(x, t)
$$

$\left(H_{3}^{\prime}\right)$ There exists positive constant $\gamma_{j}$, such that

$$
y_{j}(x, t) \beta_{j}\left(y_{j}(x, t)\right) \geq \gamma_{j} y_{j}^{2}(x, t)
$$

$\left(H_{4}\right)$ There are nonnegative functions $c_{i j}^{0}(t), c_{i j}^{1}(t)$, for all $t, u, v \in R$, such that

$$
\sigma_{i j}^{2}(t, u, v) \leq c_{i j}^{0}(t) u^{2}+c_{i j}^{1}(t) v^{2}
$$

$\left(H_{4}^{\prime}\right)$ There are nonnegative constants $c_{i j}^{0}, c_{i j}^{1}$, for all $t, u, v \in R$, such that

$$
\sigma_{i j}^{2}(t, u, v) \leq c_{i j}^{0} u^{2}+c_{i j}^{1} v^{2}
$$

$\left(H_{5}\right)$ The following inequality holds:

$$
\int_{\Omega} D_{i k}\left(\frac{\partial y_{i}}{\partial x_{k}}\right)_{k=1}^{m} \cdot \nabla y_{i}^{p-1} \mathrm{~d} x>0
$$


Definition 2.1. The trivial solution of (1.4) is said to be $p$ th moment exponentially stable if there is a pair of positive constants $\lambda$ and $G$ such that

$$
E\|y(\phi, t)\|^{p}<G E\|\phi\|^{p} e^{-\lambda\left(t-t_{0}\right)}, \quad \text { on } t \geq t_{0}
$$

for any $\phi$, where $\lambda$ also called as convergence rate. When $p=2$, it is usually said to be exponentially stable in mean square.

Definition 2.2. Let $h: R \rightarrow R$ be a continuous function, $d^{+} h$; the upper right Dini-derivative of $h$ is defined as

$$
d^{+} h(t)=\limsup _{\delta \rightarrow 0^{+}} \frac{h(t+\delta)-h(t)}{\delta}
$$

The following lemmas are important in our approach.

Lemma 2.3 (Hardy inequality [4]). Assume there exist constants $a_{k} \geq 0, p_{k}>0(k=1, \ldots, m+$ 1), then the following inequality holds:

$$
\left(\prod_{k=1}^{m+1} a_{k}^{p_{k}}\right)^{1 / S_{m+1}} \leq\left(\sum_{k=1}^{m+1} p_{k} a_{k}^{r}\right)^{1 / r} S_{m+1}^{-1 / r},
$$

where $r>0$ and $S_{m+1}=\sum_{k=1}^{m+1} p_{k}$. In (2.11), if one lets $p_{m+1}=1, r=S_{m+1}=\sum_{k=1}^{m} p_{k}+1$, one will get

$$
\left(\prod_{k=1}^{m} a_{k}^{p_{k}}\right) a_{m+1} \leq \frac{1}{r}\left(\sum_{k=1}^{m} p_{k} a_{k}^{r}\right)+\frac{1}{r} a_{m+1}^{r},
$$

if one lets $p_{m+1}=2, r=S_{m+1}=\sum_{k=1}^{m} p_{k}+2$, one will get

$$
\left(\prod_{k=1}^{m} a_{k}^{p_{k}}\right) a_{m+1} \leq \frac{1}{r}\left(\sum_{k=1}^{m} p_{k} a_{k}^{r}\right)+\frac{2}{r} a_{m+1}^{r} .
$$

Lemma 2.4 (generalized Halanay inequality [37]). For two positive-valued functions $a(t)$ and $b(t)$ defined on $\left[t_{0} \infty\right)$, assume there exists a constant number $0 \leq \mu<1$ satisfying $0<a_{0} \leq a(t), 0<$ $b(t) \leq \mu a(t)$ hold for all $t \geq t_{0} ; y(t)$ is nonnegative continuous function on $\left[t_{0}-\tau, \infty\right)$ and satisfies the following inequality:

$$
d^{+} y(t) \leq-a(t) y(t)+b(t) \bar{y}(t) \quad \text { for } t \geq t_{0}
$$

where $\bar{y}(t)=\sup _{t-\tau \leq s \leq t} y(s) ; \tau \geq 0$ is constant. Then one has

$$
y(t) \leq \bar{y}\left(t_{0}\right) e^{-\lambda^{*}\left(t-t_{0}\right)},
$$


where $\lambda^{*}>0$ is defined as

$$
\lambda^{*}=\inf _{t \geq t_{0}}\left\{\lambda(t): \lambda=a(t)-b(t) e^{\lambda \tau}\right\}
$$

\section{Main Results}

Theorem 3.1. Under assumptions $\left(H_{1}\right),\left(H_{2}\right),\left(H_{3}\right),\left(H_{4}\right),\left(H_{5}\right)$, if there exist a positive diagonal matrix $Q=\operatorname{diag}\left(q_{1}, \ldots, q_{n}\right)$, constants $v_{k}>0\left(k=1, \ldots, k_{1}\right), \mu_{k}>0\left(k=1, \ldots, k_{2}\right), \rho_{k}>0(k=$ $\left.1, \ldots, k_{3}\right), \xi_{i j}, \xi_{i j}^{*}, \eta_{i j}, \eta_{i j}^{*}, m_{i j}, m_{i j}^{*}, p_{i j}, p_{i j}^{*}, q_{i j}, q_{i j}^{*} \in \mathbb{R}, 0<\lambda_{2}$, and $0 \leq \mu<1$, such that

$$
0<\lambda_{2} \leq \lambda_{2}(t) \leq \mu \lambda_{1}(t), \text { holds for all } t \geq t_{0},
$$

where

$$
\begin{gathered}
\lambda_{1}(t)=\min _{1 \leq i \leq n}\left\{\underline{p} \underline{\alpha_{i}} \gamma_{i}(t)-\sum_{j=1}^{n} \overline{\alpha_{i}} \sum_{k=1}^{k_{1}} v_{k}\left|a_{i j}(t)\right|^{p \xi_{i j} / v_{k}} G_{j}^{p \eta_{i j} / v_{k}}\right. \\
-\frac{1}{q_{i}} \sum_{j=1}^{n} q_{j} \overline{\alpha_{j}}\left|a_{j i}(t)\right|^{p \xi_{j i}^{*}} G_{i}^{p \eta_{j i}^{*}}-\sum_{j=1}^{n} \overline{\alpha_{i}} \sum_{k=1}^{k_{2}} \mu_{k}\left|b_{i j}(t)\right|^{p p_{i j} / \mu_{k}} G_{j}^{p q_{i j} / \mu_{k}} \\
\left.-\frac{p(p-1)}{2} \sum_{j=1}^{n} c_{i j}^{0}(t)-\frac{p-1}{2} \sum_{j=1}^{n} \sum_{k=1}^{k_{3}} \rho_{k}\left|c_{i j}^{1}(t)\right|^{p m_{i j} / \rho_{k}}\right\}, \\
\lambda_{2}(t)=\max _{1 \leq i \leq n}\left[\sum_{j=1}^{n} \frac{q_{j}}{q_{i}} \overline{\alpha_{j}}\left|b_{j i}(t)\right|^{p p_{j i}^{*}} G_{i}^{p q_{j i}^{*}}+(p-1) \sum_{j=1}^{n} \frac{q_{j}}{q_{i}}\left(c_{j i}^{1}(t)\right)^{p m_{j i}^{*} / 2}\right]
\end{gathered}
$$

$p=\sum_{j=1}^{k_{1}} v_{k}+1=\sum_{j=1}^{k_{2}} \mu_{k}+1=\sum_{j=1}^{k_{3}} \rho_{k}+2, k_{1} \xi_{i j}+\xi_{i j}^{*}=1, k_{1} \eta_{i j}+\eta_{i j}^{*}=1, k_{2} p_{i j}+p_{i j}^{*}=1, k_{2} q_{i j}+q_{i j}^{*}=1$, $k_{3} m_{i j}+m_{i j}^{*}=1, k_{3} m_{i j}+m_{i j}^{*}=1$, then for all $\xi \in L_{q_{0}}^{p}\left([-\tau, 0], R^{n}\right)$, the trivial solution of system (1.4) is pth moment exponentially stable, where $p \geq 2$ is a constant.

Proof. Consider the following Lyapunov function:

$$
V(t, y(t))=\int_{\Omega} \sum_{i=1}^{n} q_{i}\left|y_{i}(x, t)\right|^{p} \mathrm{~d} x
$$


Applying Itô formula to $V(t, y(t))$, for $\delta>0$, we can get

$$
\begin{aligned}
& V(t+\delta, y(t+\delta))-V(t, y(t)) \\
& =\int_{t}^{t+\delta} V_{t}(s, y(s)) \mathrm{d} s \\
& +\int_{t}^{t+\delta} V_{y}(s, y(s))\left\{\sum_{k=1}^{m} \frac{\partial}{\partial x_{k}}\left(D_{i k} \frac{\partial y_{i}}{\partial x_{k}}\right)-\alpha_{i}\left(y_{i}(x, s)\right)\left[\beta_{i}\left(y_{i}(x, s)\right)-\sum_{j=1}^{n} a_{i j}(s) g_{j}\left(y_{j}(x, s)\right)\right.\right. \\
& \left.\left.-\sum_{j=1}^{n} b_{i j}(s) g_{j}\left(y_{j}\left(x, s-\tau_{j}(s)\right)\right)\right]\right\} \mathrm{d} s \\
& +\int_{t}^{t+\delta} V_{y}(s, y(s)) \sum_{j=1}^{n} \sigma_{i j}\left(s, y_{i}(x, s), y_{j}\left(x, s-\tau_{j}(s)\right)\right) \mathrm{d} \omega_{j}(s)+\int_{t}^{t+\delta} \frac{1}{2} \operatorname{trace}\left(\sigma^{T} V_{y y} \sigma\right) \mathrm{d} s \\
& \leq \int_{t}^{t+\delta} \int_{\Omega} p \sum_{i=1}^{n} q_{i}\left|y_{i}^{p-2}(x, s)\right| y_{i}(x, s) \\
& \times\left\{\sum_{k=1}^{m} \frac{\partial}{\partial x_{k}}\left(D_{i k} \frac{\partial y_{i}}{\partial x_{k}}\right)-\alpha_{i}\left(y_{i}(x, s)\right)\left[\beta_{i}\left(y_{i}(x, s)\right)-\sum_{j=1}^{n} a_{i j}(s) g_{j}\left(y_{j}(x, s)\right)\right.\right. \\
& \left.\left.-\sum_{j=1}^{n} b_{i j}(s) g_{j}\left(y_{j}\left(x, s-\tau_{j}(s)\right)\right)\right]\right\} \mathrm{d} x \mathrm{~d} s \\
& +\int_{t}^{t+\delta} V_{y}(s, y(s)) \sum_{j=1}^{n} \sigma_{i j}\left(s, y_{i}(x, s), y_{j}\left(x, s-\tau_{j}(s)\right)\right) \mathrm{d} \omega_{j}(s)+\int_{t}^{t+\delta} \frac{1}{2} \operatorname{trace}\left(\sigma^{T} V_{y y} \sigma\right) \mathrm{d} s \\
& \leq \int_{t}^{t+\delta} \int_{\Omega} p\left\{\sum_{i=1}^{n} q_{i}\left|y_{i}^{p-1}(x, s)\right| \sum_{k=1}^{m} \frac{\partial}{\partial x_{k}}\left(D_{i k} \frac{\partial y_{i}}{\partial x_{k}}\right)-\sum_{i=1}^{n} q_{i} \alpha_{i} \gamma_{i}(s)\left|y_{i}^{p}(x, s)\right|\right. \\
& +\sum_{i=1}^{n} \sum_{j=1}^{n} q_{i} \bar{\alpha}_{i} G_{j}\left|a_{i j}(s) y_{i}^{p-1}(x, s) y_{j}(x, s)\right| \\
& \left.+\sum_{i=1}^{n} \sum_{j=1}^{n} q_{i} \overline{\alpha_{i}} G_{j}\left|b_{i j}(s) y_{i}^{p-1}(x, s) y_{j}\left(x, s-\tau_{j}(s)\right)\right|\right\} \mathrm{d} x \mathrm{~d} s \\
& +p \int_{t}^{t+\delta} \int_{\Omega} \sum_{i=1}^{n} \sum_{j=1}^{n} q_{i}\left|y_{i}^{p-1}(x, s)\right| \sigma_{i j}\left(s, y_{i}(x, s), y_{j}\left(x, s-\tau_{j}(s)\right)\right) \mathrm{d} x \mathrm{~d} \omega_{j}(s) \\
& +\int_{t}^{t+\delta} \int_{\Omega} \frac{p(p-1)}{2} q_{i} \sum_{i=1}^{n} \sum_{j=1}^{n}\left[c_{i j}^{0}(s)\left|y_{i}^{p}(x, s)\right|+c_{i j}^{1}(s)\left|y_{i}^{p-2}(x, s) y_{j}^{2}\left(x, s-\tau_{j}(s)\right)\right|\right] \mathrm{d} x \mathrm{~d} s .
\end{aligned}
$$


From the boundary condition, we get

$$
\begin{aligned}
& \sum_{k=1}^{m} \int_{\Omega} y_{i}^{p-1} \frac{\partial}{\partial x_{k}}\left(D_{i k} \frac{\partial y_{i}}{\partial x_{k}}\right) \mathrm{d} x \\
& \quad=\int_{\Omega} y_{i}^{p-1} \nabla \cdot\left(D_{i k} \frac{\partial y_{i}}{\partial x_{k}}\right)_{k=1}^{m} \mathrm{~d} x \\
& \quad=\int_{\Omega} \nabla \cdot\left(y_{i}^{p-1} D_{i k} \frac{\partial y_{i}}{\partial x_{k}}\right)_{k=1}^{m} \mathrm{~d} x-\int_{\Omega}\left(D_{i k} \frac{\partial y_{i}}{\partial x_{k}}\right)_{k=1}^{m} \cdot \nabla y_{i}^{p-1} \mathrm{~d} x \\
&=\int_{\partial \Omega}\left(\left(y_{i}^{p-1} D_{i k} \frac{\partial y_{i}}{\partial x_{k}}\right)\right)_{k=1}^{m} \cdot \mathrm{d} S-\int_{\Omega}\left(D_{i k} \frac{\partial y_{i}}{\partial x_{k}}\right)_{k=1}^{m} \cdot \nabla y_{i}^{p-1} \mathrm{~d} x \\
&=-\int_{\Omega}\left(D_{i k} \frac{\partial y_{i}}{\partial x_{k}}\right)_{k=1}^{m} \cdot \nabla y_{i}^{p-1} \mathrm{~d} x,
\end{aligned}
$$

in which, $\nabla=\left(\partial / \partial x_{1}, \ldots, \partial / \partial x_{m}\right)^{T}$ is the gradient operator, and

$$
\left(D_{i k} \frac{\partial y_{i}}{\partial x_{k}}\right)_{k=1}^{m}=\left(D_{i 1} \frac{\partial y_{i}}{\partial x_{1}}, \ldots, D_{i m} \frac{\partial y_{i}}{\partial x_{m}}\right)^{T}
$$

On the other hand, from Lemma 2.3, we have

$$
\begin{aligned}
& p G_{j}\left|a_{i j}(s) y_{i}^{p-1}(x, s) y_{j}(x, s)\right| \\
& \quad=p \prod_{k=1}^{k_{1}}\left(\left|a_{i j}(s)\right|^{\xi_{i j} / v_{k}} G_{j}^{\eta_{i j} / v_{k}}\left|y_{i}(x, s)\right|\right)^{v_{k}}\left|a_{i j}(s)\right|^{\xi_{i j}^{*}} G_{j}^{\eta_{i j}^{*}}\left|y_{j}(x, s)\right| \\
& \quad \leq \sum_{k=1}^{k_{1}} v_{k}\left|a_{i j}(s)\right|^{p \xi_{i j} / v_{k}} G_{j}^{p \eta_{i j} / v_{k}}\left|y_{i}(x, s)\right|^{p}+\left|a_{i j}(s)\right|^{p \xi_{i j}^{*}} G_{j}^{p \eta_{i j}^{*}}\left|y_{j}(x, s)\right|^{p}, \\
& p G_{j}\left|b_{i j}(s) y_{i}^{p-1}(x, s) y_{j}\left(x, s-\tau_{j}(s)\right)\right| \\
& \quad=p \prod_{k=1}^{k_{2}}\left(\left|b_{i j}(s)\right|^{p_{i j} / \mu_{k}} G_{j}^{q_{i j} / \mu_{k}}\left|y_{i}(x, s)\right|\right)^{\mu_{k}}\left|b_{i j}(s)\right|^{p_{i j}^{*}} G_{j}^{q_{i j}^{*}}\left|y_{j}\left(x, s-\tau_{j}(s)\right)\right| \\
& \leq \sum_{k=1}^{k_{2}} \mu_{k}\left|b_{i j}(s)\right|^{p p_{i j} / \mu_{k}} G_{j}^{p q_{i j} / \mu_{k}}\left|y_{i}(x, s)\right|^{p}+\left|b_{i j}(s)\right|^{p p_{i j}^{*}} G_{j}^{p q_{i j}^{*}}\left|y_{j}\left(x, s-\tau_{j}(s)\right)\right|^{p}, \\
& \frac{p(p-1)}{2} c_{i j}^{1}(s)\left|y_{i}^{p-2}(x, s) y_{j}^{2}\left(x, s-\tau_{j}(s)\right)\right| \\
& \quad=\frac{p(p-1)}{2} \prod_{k=1}^{k_{3}}\left(\left|c_{i j}^{1}(s)\right|^{m_{i j} / \rho_{k}}\left|y_{i}(x, s)\right|\right)^{\rho_{k}}\left(\left|c_{i j}^{1}(s)\right|^{m_{i j}^{*} / 2}\left|y_{j}\left(x, s-\tau_{j}(s)\right)\right|\right)^{2} \\
& \leq \frac{p-1}{2} \sum_{k=1}^{k_{3}} \rho_{k}\left|c_{i j}^{1}(s)\right|^{p m_{i j} / \rho_{k}}\left|y_{i}(x, s)\right|^{p}+(p-1)\left|c_{i j}^{1}(s)\right|^{p m_{i j}^{*} / 2}\left|y_{j}\left(x, s-\tau_{j}(s)\right)\right|^{p} .
\end{aligned}
$$


It follows from (3.4), (3.5), (3.7), (3.8), and (3.9) that

$$
\begin{aligned}
& V(t+\delta, y(t+\delta))-V(t, y(t)) \\
& \leq \int_{t}^{t+\delta} \int_{\Omega}\left\{-\sum_{i=1}^{n} q_{i} p \underline{\alpha_{i}} \gamma_{i}(s)\left|y_{i}(x, s)\right|^{p}\right. \\
& +\sum_{i=1}^{n} \sum_{j=1}^{n} q_{i} \overline{\alpha_{i}}\left[\sum_{k=1}^{k_{1}} v_{k}\left|a_{i j}(s)\right|^{p \xi_{i j} / v_{k}} G_{j}^{p \eta_{i j} / v_{k}}\left|y_{i}(x, s)\right|^{p}+\left|a_{i j}(s)\right|^{p \xi_{i j}^{*}} G_{j}^{p \eta_{i j}^{*}}\left|y_{j}(x, s)\right|^{p}\right] \\
& +\sum_{i=1}^{n} \sum_{j=1}^{n} q_{i} \overline{\alpha_{i}}\left[\sum_{k=1}^{k_{2}} \mu_{k}\left|b_{i j}(s)\right|^{p p_{i j} / \mu_{k}} G_{j}^{p q_{i j} / \mu_{k}}\left|y_{i}(x, s)\right|^{p}\right. \\
& \left.\left.+\left|b_{i j}(s)\right|^{p p_{i j}^{*}} G_{j}^{p q_{i j}^{*}}\left|y_{j}\left(x, s-\tau_{j}(s)\right)\right|^{p}\right]\right\} \mathrm{d} x \mathrm{~d} s \\
& +p \int_{t}^{t+\delta} \int_{\Omega} \sum_{i=1}^{n} \sum_{j=1}^{n} q_{i}\left|y_{i}(x, s)\right|^{p-1} \sigma_{i j}\left(s, y_{i}(x, s), y_{j}\left(x, s-\tau_{j}(s)\right)\right) \mathrm{d} x \mathrm{~d} \omega_{j}(s) \\
& +\int_{t}^{t+\delta} \int_{\Omega} q_{i}\left\{\sum_{i=1}^{n} \sum_{j=1}^{n} \frac{p(p-1)}{2} c_{i j}^{0}(s)\left|y_{i}(x, s)\right|^{p}\right. \\
& +\sum_{i=1}^{n} \sum_{j=1}^{n}\left[\frac{p-1}{2} \sum_{k=1}^{k_{3}} \rho_{k}\left|c_{i j}^{1}(s)\right|^{p m_{i j} / \rho_{k}}\left|y_{i}(x, s)\right|^{p}\right. \\
& \left.\left.+(p-1)\left|c_{i j}^{1}(s)\right|^{p m_{i j}^{*} / 2}\left|y_{j}\left(x, s-\tau_{j}(s)\right)\right|^{p}\right]\right\} \mathrm{d} x \mathrm{~d} s \\
& \leq \int_{t}^{t+\delta}-\min _{1 \leq i \leq n}\left\{p \underline{\alpha_{i}} \gamma_{i}(s)-\sum_{j=1}^{n} \overline{\alpha_{i}} \sum_{k=1}^{k_{1}} v_{k}\left|a_{i j}(s)\right|^{p \xi_{i j} / v_{k}} G_{j}^{p \eta_{i j} / v_{k}}-\sum_{j=1}^{n} \frac{q_{j}}{q_{i}} \overline{\alpha_{j}}\left|a_{j i}(s)\right|^{p \xi_{j i}^{*}} G_{i}^{p \eta_{j i}^{*}}\right. \\
& -\sum_{j=1}^{n} \overline{\alpha_{i}} \sum_{k=1}^{k_{2}} \mu_{k}\left|b_{i j}(s)\right|^{p p_{i j} / \mu_{k}} G_{j}^{p q_{i j} / \mu_{k}}-\frac{p(p-1)}{2} \sum_{j=1}^{n} c_{i j}^{0}(s) \\
& \left.-\frac{p-1}{2} \sum_{j=1}^{n} \sum_{k=1}^{k_{3}} \rho_{k}\left|c_{i j}^{1}(s)\right|^{p m_{i j} / \rho_{k}}\right\} V(s, y(s)) \mathrm{d} s \\
& +p \int_{t}^{t+\delta} \int_{\Omega} \sum_{i=1}^{n} \sum_{j=1}^{n} q_{i}\left|y_{i}(x, s)\right|^{p-1} \sigma_{i j}\left(s, y_{i}(x, s), y_{j}\left(x, s-\tau_{j}(s)\right)\right) \mathrm{d} x \mathrm{~d} \omega_{j}(s) \\
& +\int_{t}^{t+\delta} \max _{1 \leq i \leq n}\left\{\sum_{j=1}^{n} \frac{q_{j}}{q_{i}} \overline{\alpha_{j}}\left|b_{j i}(s)\right|^{p p_{j i}^{*}} G_{i}^{p q_{j i}^{*}}+(p-1) \sum_{j=1}^{n} \frac{q_{j}}{q_{i}}\left(c_{j i}^{1}(s)\right)^{p m_{j i}^{*} / 2}\right\} V(s-\tau(s), y(s-\tau(s))) \mathrm{d} s .
\end{aligned}
$$


By [38, Theorem 4.2.8], we know that

$$
E \int_{t}^{t+\delta} \int_{\Omega} \sum_{i=1}^{n} \sum_{j=1}^{n} q_{i}\left|y_{i}(x, s)\right|^{p-1} \sigma_{i j}\left(s, y_{i}(x, s), y_{j}\left(x, s-\tau_{j}(s)\right)\right) \mathrm{d} x \mathrm{~d} \omega_{j}(s)=0 .
$$

Therefore, taking expectation on both sides of (3.10), the preceding result leads directly to

$$
\begin{aligned}
& E V(t+\delta, y(t+\delta))-E V(t, y(t)) \\
& \left.\begin{array}{l}
\leq \int_{t}^{t+\delta}-\min _{1 \leq i \leq n}\left\{p \underline{\alpha_{i}} \gamma_{i}(s)-\sum_{j=1}^{n} \overline{\alpha_{i}} \sum_{k=1}^{k_{1}} v_{k}\left|a_{i j}(\mathrm{~s})\right|^{p \xi_{i j} / v_{k}} G_{j}^{p \eta_{i j} / v_{k}}\right. \\
-\sum_{j=1}^{n} \frac{q_{j}}{q_{i}} \overline{\alpha_{j}}\left|a_{j i}(s)\right|^{p \xi_{j i}^{*}} G_{i}^{p \eta_{j i}^{*}}-\sum_{j=1}^{n} \bar{\alpha}_{i} \sum_{k=1}^{k_{2}} \mu_{k}\left|b_{i j}(s)\right|^{p p_{i j} / \mu_{k}} G_{j}^{p q_{i j} / \mu_{k}} \\
\left.-\frac{p(p-1)}{2} \sum_{j=1}^{n} c_{i j}^{0}(s)-\frac{p-1}{2} \sum_{j=1}^{n} \sum_{k=1}^{k_{3}} \rho_{k}\left|c_{i j}^{1}(s)\right|^{p m_{i j} / \rho_{k}}\right\} E V(s, y(s)) \mathrm{d} s \\
+\int_{t}^{t+\delta} \max _{1 \leq i \leq n}\left\{\sum_{j=1}^{n} \frac{q_{j}}{q_{i}} \overline{\alpha_{j}}\left|b_{j i}(s)\right|^{p p_{j i}^{*}} G_{i}^{p q_{j i}^{*}}+(p-1) \sum_{j=1}^{n} \frac{q_{j}}{q_{i}}\left(c_{j i}^{1}(s)\right)^{p m_{j i}^{*} / 2}\right\} \\
\times E V(s-\tau(s), y(s-\tau(s))) \mathrm{d} s .
\end{array}\right\}
\end{aligned}
$$

By the mean value theorem for integrals, we have

$$
d^{+} E V(t, y(t)) \leq-\lambda_{1}(t) E V(t, y(t))+\lambda_{2}(t) \sup _{t-\tau \leq s \leq t} E V(s, y(s))
$$

By Lemma 2.4, we get

$$
E V(t, y(t)) \leq \sup _{t_{0}-\tau \leq s \leq t_{0}} E V(s, y(s)) e^{-\lambda^{*}\left(t-t_{0}\right)}
$$

where $\lambda^{*}>0$ is defined as

$$
\lambda^{*}=\inf _{t \geq t_{0}}\left\{\lambda(t): \lambda=\lambda_{1}(t)-\lambda_{2}(t) e^{\lambda \tau}\right\} .
$$


That is to say

$$
E\|y(\phi, t)\|^{p}<G E\|\phi\|^{p} e^{-\lambda^{*}\left(t-t_{0}\right)}, \quad \text { on } t \geq t_{0},
$$

where

$$
G=\frac{\max _{1 \leq i \leq n}\left\{m_{i}\right\}}{\min _{1 \leq i \leq n}\left\{m_{i}\right\}}
$$

Therefore, the trivial solution of system (1.4) is $p$ th moment exponentially stable. Furthermore, just as discussed in [39, pp. 173-180], the trivial solution of (1.4) is also almost surely exponentially stable. Theorem 3.1 also shows that the reaction-diffusion term has no influence on the stability for system (1.4).

In Theorem 3.1, if we take $k_{1}=k_{2}=k_{3}=1, v_{k}=\mu_{k}=p-1, \rho_{k}=p-2, \xi_{i j}=\eta_{i j}=p_{i j}=$ $q_{i j}=(p-1) / p, m_{i j}=\mu_{k}=(p-2) / p, \xi_{i j}^{*}=\eta_{i j}^{*}=p_{i j}^{*}=q_{i j}^{*}=1 / p, m_{i j}^{*}=\mu_{k}=2 / p$, we have the following result.

Corollary 3.2. Under assumptions $\left(H_{1}\right),\left(H_{2}\right),\left(H_{3}\right),\left(H_{4}\right),\left(H_{5}\right)$, if there exist a positive diagonal matrix $Q=\operatorname{diag}\left(q_{1}, \ldots, q_{n}\right), 0<\lambda_{2}$, and $0 \leq \mu<1$, such that

$$
0<\lambda_{2} \leq \lambda_{2}(t) \leq \mu \lambda_{1}(t), \quad \text { holds for all } t \geq t_{0},
$$

where

$$
\begin{gathered}
\lambda_{1}(t)=\min _{1 \leq i \leq n}\left\{\underline{p} \underline{\alpha_{i}} \gamma_{i}(t)-(p-1) \sum_{j=1}^{n} \overline{\alpha_{i}}\left|a_{i j}(t)\right| G_{j}\right. \\
-\frac{1}{q_{i}} \sum_{j=1}^{n} \overline{\alpha_{j}} q_{j}\left|a_{j i}(t)\right| G_{i}-(p-1) \sum_{j=1}^{n} \overline{\alpha_{i}}\left|b_{i j}(t)\right| G_{j} \\
\left.-\frac{p(p-1)}{2} \sum_{j=1}^{n} c_{i j}^{0}(t)-\frac{(p-1)(p-2)}{2} \sum_{j=1}^{n} c_{i j}^{1}(t)\right\}, \\
\lambda_{2}(t)=\max _{1 \leq i \leq n}\left[\sum_{j=1}^{n} \frac{q_{j}}{q_{i}}\left|b_{j i}(t)\right| \overline{\alpha_{j}} G_{i}+(p-1) \sum_{j=1}^{n} \frac{q_{j}}{q_{i}} c_{j i}^{1}(t)\right],
\end{gathered}
$$

then for all $\xi \in L_{\mathcal{q}_{0}}^{p}\left([-\tau, 0], R^{n}\right)$, the trivial solution of system (1.4) is pth moment exponentially stable, where $p \geq 2$ is a constant. 
When $a_{i j}(t) \equiv a_{i j}, b_{i j}(t) \equiv b_{i j}$, model (1.4) is reduced to the following stochastic reaction-diffusion Cohen-Grossberg neural networks with time-varying delays:

$$
\begin{aligned}
\mathrm{d} y_{i}(x, t)= & \sum_{k=1}^{m} \frac{\partial}{\partial x_{k}}\left(D_{i k} \frac{\partial y_{i}}{\partial x_{k}}\right) \mathrm{d} t-\alpha_{i}\left(y_{i}(x, t)\right) \\
& \times\left[\beta_{i}\left(y_{i}(x, t)\right)-\sum_{j=1}^{n} a_{i j} g_{j}\left(y_{j}(x, t)\right)-\sum_{j=1}^{n} b_{i j} g_{j}\left(y_{j}\left(x, t-\tau_{j}(t)\right)\right)\right] \mathrm{d} t \\
& +\sum_{j=1}^{n} \sigma_{i j}\left(t, y_{i}(x, t), y_{j}\left(x, t-\tau_{j}(t)\right)\right) \mathrm{d} \omega_{j}(t),
\end{aligned}
$$

Corollary 3.3. Under assumptions $\left(H_{1}\right),\left(H_{2}\right),\left(H_{3}^{\prime}\right),\left(H_{4}^{\prime}\right),\left(H_{5}\right)$, if there exist a positive diagonal matrix $Q=\operatorname{diag}\left(q_{1}, \ldots, q_{n}\right)$, constants $v_{k}>0\left(k=1, \ldots, k_{1}\right), \mu_{k}>0\left(k=1, \ldots, k_{2}\right), \rho_{k}>0(k=$ $\left.1, \ldots, k_{3}\right), \xi_{i j}, \xi_{i j}^{*}, \eta_{i j}, \eta_{i j}^{*}, m_{i j}, m_{i j}^{*}, p_{i j}, p_{i j}^{*}, q_{i j}, q_{i j}^{*} \in \mathbb{R}$, such that

$$
\lambda_{2}<\lambda_{1}
$$

where

$$
\begin{aligned}
& \lambda_{1}=\min _{1 \leq i \leq n}\{ p \underline{\alpha_{i}} \gamma_{i}-\sum_{j=1}^{n} \overline{\alpha_{i}} \sum_{k=1}^{k_{1}} v_{k}\left|a_{i j}\right|^{p \xi_{i j} / v_{k}} G_{j}^{p \eta_{i j} / v_{k}}-\frac{1}{q_{i}} \sum_{j=1}^{n} q_{j} \overline{\alpha_{j}}\left|a_{j i}\right|^{p \xi_{j i}^{*}} G_{i}^{p \eta_{j i}^{*}} \\
&\left.\quad-\sum_{j=1}^{n} \overline{\alpha_{i}} \sum_{k=1}^{k_{2}} \mu_{k}\left|b_{i j}\right|^{p p_{i j} / \mu_{k}} G_{j}^{p q_{i j} / \mu_{k}}-\frac{p(p-1)}{2} \sum_{j=1}^{n} c_{i j}^{0}-\frac{p-1}{2} \sum_{j=1}^{n} \sum_{k=1}^{k_{3}} \rho_{k}\left|c_{i j}^{1}\right|^{p m_{i j} / \rho_{k}}\right\}, \\
& \lambda_{2}=\max _{1 \leq i \leq n}\left[\sum_{j=1}^{n} \frac{q_{j}}{q_{i}} \overline{\alpha_{j}}\left|b_{j i}\right|^{p p_{j i}^{*}} G_{i}^{p q_{j i}^{*}}+(p-1) \sum_{j=1}^{n} \frac{q_{j}}{q_{i}}\left(c_{j i}^{1}\right)^{p m_{j i}^{*} / 2}\right]
\end{aligned}
$$

$p=\sum_{j=1}^{k_{1}} v_{k}+1=\sum_{j=1}^{k_{2}} \mu_{k}+1=\sum_{j=1}^{k_{3}} \rho_{k}+2, k_{1} \xi_{i j}+\xi_{i j}^{*}=1, k_{1} \eta_{i j}+\eta_{i j}^{*}=1, k_{2} p_{i j}+p_{i j}^{*}=1, k_{2} q_{i j}+q_{i j}^{*}=1$, $k_{3} m_{i j}+m_{i j}^{*}=1, k_{3} m_{i j}+m_{i j}^{*}=1$, then for all $\xi \in L_{\mathcal{F}_{0}}^{p}\left([-\tau, 0], R^{n}\right)$, the trivial solution of system (3.20) is pth moment exponentially stable, where $p \geq 2$ is a constant.

In Corollary 3.3, if we take $k_{1}=k_{2}=k_{3}=1, v_{k}=\mu_{k}=p-1, \rho_{k}=p-2, \xi_{i j}=\eta_{i j}=p_{i j}=$ $q_{i j}=(p-1) / p, m_{i j}=\mu_{k}=(p-2) / p, \xi_{i j}^{*}=\eta_{i j}^{*}=p_{i j}^{*}=q_{i j}^{*}=1 / p, m_{i j}^{*}=\mu_{k}=2 / p$, we have the following result. 
Corollary 3.4. Under assumptions $\left(H_{1}\right),\left(H_{2}\right),\left(H_{3}^{\prime}\right),\left(H_{4}^{\prime}\right),\left(H_{5}\right)$, if there exists a positive diagonal matrix $Q=\operatorname{diag}\left(q_{1}, \ldots, q_{n}\right)$, such that

$$
\lambda_{1}>\lambda_{2}
$$

where

$$
\begin{aligned}
\lambda_{1}=\min _{1 \leq i \leq n}\left\{\underline{p} \underline{\alpha_{i}} \gamma_{i}-(p-1) \sum_{j=1}^{n} \overline{\alpha_{i}}\left|a_{i j}\right| G_{j}-\frac{1}{q_{i}} \sum_{j=1}^{n} \overline{\alpha_{j}} q_{j}\left|a_{j i}\right| G_{i}\right. \\
\left.\quad-(p-1) \sum_{j=1}^{n} \overline{\alpha_{i}}\left|b_{i j}\right| G_{j}-\frac{p(p-1)}{2} \sum_{j=1}^{n} c_{i j}^{0}-\frac{(p-1)(p-2)}{2} \sum_{j=1}^{n} c_{i j}^{1}\right\}, \\
\lambda_{2=} \max _{1 \leq i \leq n}\left[\frac{1}{q_{i}} \sum_{j=1}^{n} q_{j}\left|b_{j i}\right| \overline{\alpha_{j}} G_{i}+(p-1) \frac{1}{q_{i}} \sum_{j=1}^{n} q_{j} c_{j i}^{1}\right],
\end{aligned}
$$

then for all $\xi \in L_{\mp_{0}}^{p}\left([-\tau, 0], R^{n}\right)$, the trivial solution of system (3.20) is pth moment exponentially stable.

Remark 3.5. Model (3.20) has been studied in [40] and the main results in [40, Theorem 1, Corollaries 1 and 2] are the direct results of Corollary 3.4 in our paper when we choose $p=2$.

Remark 3.6. When $D_{i k}=0(i=1, n, k=i, \ldots, m)$ system (3.20) is reduced to the stochastic Cohen-Grossberg neural networks (1.3), which has been studied in [24, 28]. Unfortunately, the assumed condition $\left(A_{2}\right)$ in [28] is not correct, a defect appearing in the main result in [28] when $p=2 k+1, k \in Z^{+}, x(t)<0$, just from the constructed Lyapunov function, one can find that the term " $x^{p / 2}(t)$ " is a blemish. The constructed Lyapunov function should be replaced with $V(t, x(t))=\sum_{i=1}^{n} q_{i}\left|x_{i}(t)\right|^{p}$. Noticing that $\partial\left|x_{i}(t)\right|^{p} / \partial x_{i}=p\left|x_{i}\right|^{p-1} \operatorname{sgn}\left\{x_{i}\right\}=p\left|x_{i}\right|^{p-2} x_{i}$, we have $\left(\partial\left|x_{i}(t)\right|^{p} / \partial x_{i}\right) \beta_{i}\left(x_{i}(t)\right)=p\left|x_{i}\right|^{p-2} x_{i} \beta_{i}\left(x_{i}(t)\right)$, so the assumed condition $\left(A_{2}\right)$ should be revised as $\left(H_{3}^{\prime}\right)$. On the other hand, there is an error appear in (1.3), the coefficient of $\lambda_{1}$, the term " $(p-1)(p-2)$ " should be replaced with $((p-1)(p-2)) / 2$, therefore, the main results obtained in [28] are somewhat errors. Obviously, Theorem 3.1 in our paper modifies and generalizes the main results in [28] greatly. Just choosing $p=2$, one can get a set of corollary easily, which also generalizes the main results in [24].

Remark 3.7. When $D_{i k}=0(i=1, n, k=i, \ldots, m), \sigma_{i j}(t, \cdot, \cdot)=0$, system (1.4) is reduced to a deterministic Cohen-Grossberg neural networks with time-varying delays model, just choosing some special parameters, using Theorem 3.1, one can get a set of corollary easily, which also generalizes some corresponding results in [4].

\section{An Illustrative Example}

In this section, a numerical example is presented to illustrate the correctness of our main result. 
Example 4.1. Consider a two-dimensional stochastic reaction-diffusion Cohen-Grossberg neural networks with time-varying delays as follows:

$$
\begin{aligned}
& \mathrm{d} y(t)=\left(\begin{array}{c}
\sum_{k=1}^{2} \frac{\partial}{\partial x_{k}}\left(D_{1 k} \frac{\partial y_{1}}{\partial x_{k}}\right) \\
\sum_{k=1}^{2} \frac{\partial}{\partial x_{k}}\left(D_{2 k} \frac{\partial y_{2}}{\partial x_{k}}\right)
\end{array}\right) \mathrm{d} t-\left(\begin{array}{cc}
\alpha_{1}\left(y_{1}(x, t)\right), & 0 \\
0 & \alpha_{2}\left(y_{2}(x, t)\right)
\end{array}\right) \\
& \times\left[\left(\begin{array}{cc}
\beta_{1}\left(y_{1}(x, t)\right), & 0 \\
0 & \beta_{2}\left(y_{2}(x, t)\right)
\end{array}\right)\left(\begin{array}{l}
y_{1}(t) \\
y_{2}(t)
\end{array}\right)-\left(\begin{array}{ll}
a_{11}(t), & a_{12}(t) \\
a_{21}(t), & a_{22}(t)
\end{array}\right)\left(\begin{array}{l}
\tanh \left(y_{1}(t)\right) \\
\tanh \left(y_{2}(t)\right)
\end{array}\right)\right. \\
& \left.-\left(\begin{array}{ll}
b_{11}(t), & b_{12}(t) \\
b_{21}(t), & b_{22}(t)
\end{array}\right)\left(\begin{array}{c}
\tanh \left(y_{1}\left(t-\tau_{1}(t)\right)\right) \\
\tanh \left(y_{2}\left(t-\tau_{2}(t)\right)\right)
\end{array}\right)\right] \mathrm{dt} \\
& +\sigma(t, y(t), y(t-\tau(t))) \mathrm{d} w(t), \quad t \geq 0,
\end{aligned}
$$

where $\alpha_{1}\left(y_{1}(x, t)\right)=3+\sin \left(y_{1}(x, t)\right), \alpha_{2}\left(y_{2}(x, t)\right)=3-\cos \left(y_{2}(x, t)\right), \beta_{1}\left(y_{1}(x, t)\right)=(4+$ $(3 t / 100))\left(y_{1}(x, t)\right), \beta_{2}\left(y_{2}(x, t)\right)=(5+(3 t / 100))\left(y_{2}(x, t)\right), a_{11}(t)=-1 / 8-t / 100, a_{12}(t)=$ $1 / 4, a_{21}(t)=3 / 4, a_{22}(t)=-1 / 4-(1 / 200) t, b_{11}(t)=-1 / 8-(1 / 1600) t, b_{12}(t)=1 / 4, b_{21}(t)=$ $1 / 8, b_{22}(t)=-1 / 4-(1 / 1600) t, \tau_{i}(t)$ is a bounded positive function and $\sigma: R_{+} \times R^{2} \times R^{2} \rightarrow$ $R^{2} \times R^{2}$ satisfies

$$
\operatorname{trace}\left[\sigma^{T}(t, u, v) \sigma(t, u, v)\right] \leq u_{1}^{2}+u_{2}^{2}+v_{1}^{2}+v_{2}^{2}
$$

In the example, let $p=4, D_{i k}$ be a positive constant, take $c_{i j}^{0}=c_{i j}^{1}=q_{i} \equiv 1$, by simple computation, we get

$$
\int_{\Omega} D_{i k}\left(\frac{\partial y_{i}}{\partial x_{k}}\right)_{k=1}^{2} \cdot \nabla y_{i}^{4-1} \mathrm{~d} x=\sum_{k=1}^{2} \int_{\Omega} 3 y_{i}^{2} D_{i k}\left(\frac{\partial y_{i}}{\partial x_{k}}\right)^{2} \mathrm{~d} x>0
$$

According to Corollary 3.2, one can get that

$$
\begin{aligned}
\lambda_{1}(t)=\min _{1 \leq i \leq n}\{ & p \underline{\alpha_{i}} \gamma_{i}(t)-(p-1) \sum_{j=1}^{n} \overline{\alpha_{i}}\left|a_{i j}(t)\right| G_{j} \\
& -\frac{1}{q_{i}} \sum_{j=1}^{n} \overline{\alpha_{j}} q_{j}\left|a_{j i}(t)\right| G_{i}-(p-1) \sum_{j=1}^{n} \overline{\alpha_{i}}\left|b_{i j}(t)\right| G_{j} \\
& \left.-\frac{p(p-1)}{2} \sum_{j=1}^{n} c_{i j}^{0}(t)-\frac{(p-1)(p-2)}{2} \sum_{j=1}^{n} c_{i j}^{1}(t)\right\}=\frac{23}{2}+\frac{29}{400} t,
\end{aligned}
$$




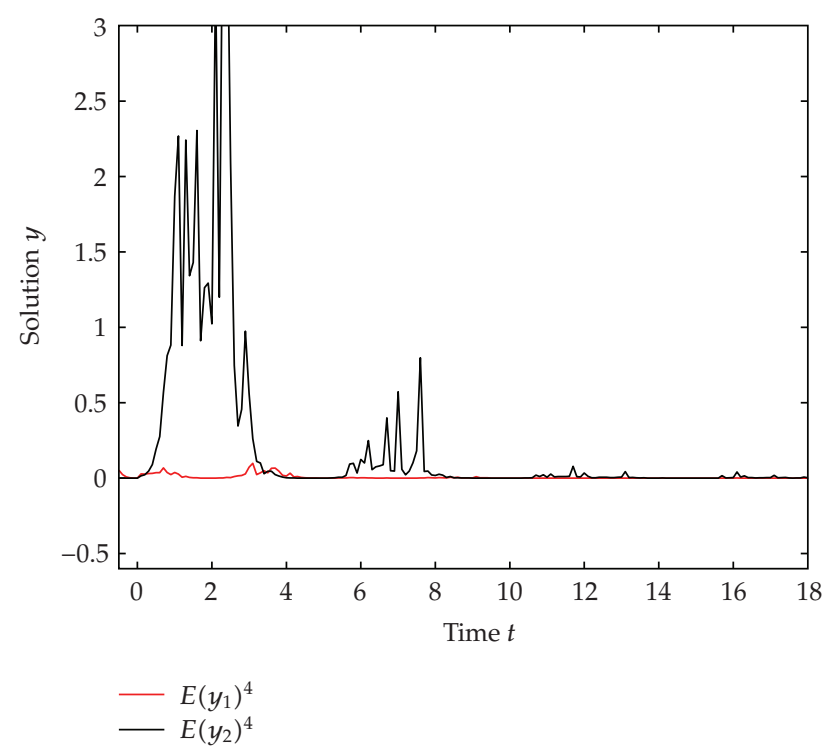

Figure 1: Numerical solution $E\left(y_{1}^{4}(t)\right), E\left(y_{2}^{4}(t)\right)$ of system (4.1).

$$
\lambda_{2}(t)=\max _{1 \leq i \leq n}\left[\sum_{j=1}^{n} \frac{q_{j}}{q_{i}}\left|b_{j i}(t)\right| \overline{\alpha_{j}} G_{i}+(p-1) \sum_{j=1}^{n} \frac{q_{j}}{q_{i}} c_{j i}^{1}(t)\right]=\frac{15}{2}+\frac{t}{400},
$$

using Corollary 3.2, system (4.1) is 4th moment exponential stable.

Remark 4.2. One can find that models considered in [20, 22-29] are special cases of model (1.4). To the best of our knowledge, few authors have considered the $p$ th moment exponential stability for Stochastic reaction-diffusion Neural Networks with time-varying connection matrix and delays. It is assumed in [22, 23, 25, 26] that delays are constants, the delay functions appear in [29] are differential and their derivatives are simultaneously required to be not greater than 1, the activation functions appear in $[22,26]$ are bounded. Obviously, we have dropped out these basic assumptions in this paper.

It is obvious that the results in [20-30] and the references therein cannot be applicable to system (4.1) even if we remove the reaction-diffusion terms from the system for the connection matrix and delays considered in this example are time-varying. This implies that the results of this paper are essentially new. Just choose $x \equiv$ constant these conclusions can be verified by the numerical simulations shown in Figure 1 .

\section{Conclusions}

In this paper, stochastic Cohen-Grossberg neural networks with time-varying delays and reaction-diffusion have been investigated. All features of stochastic systems (especially the connection matrices and delays are time-varying) reaction-diffusion systems have been taken into account in the neural networks. Without requiring the differential and monotonicity of the activation functions and the symmetry of the connection matrices, a set of new sufficient conditions for checking $p$ th moment exponential stability of the trivial solution 
of the considered system is presented by using of Lyapunov function, stochastic analysis technique, and the generalized Halanay inequality. The proposed results generalized and improved some of the earlier published results greatly. The results obtained in this paper are independent of the magnitude of delays and diffusion effect, which implies that strong selfregulation is dominant in the networks. In addition, the methods used in this paper are also applicable to other neural networks, such as stochastic Hopfield neural networks with timevarying delays and reaction-diffusion terms and stochastic bidirectional associative memory (BAM) neural networks with time-varying delays and reaction-diffusion terms. If we remove the noise and reaction-diffusion terms from the system, the derived conditions for stability of general deterministic neural networks can be viewed as byproducts of our results.

\section{Acknowledgments}

The authors are extremely grateful to two anonymous reviewers, and particularly to Professor Yong Zhou for their valuable comments and suggestions, which have contributed a lot to the improved presentation of this paper. This work was supported in part by the National Natural Science Foundation of China under Grants no. 50925727, no. 10971240, and no. 60876022, the Foundation of Chinese Society for Electrical Engineering, the Foundation of Yunnan Provincial Education Department under Grant no. 07Y10085, the Scientific Research Fund of Yunnan Province under Grant no. 2008CD186.

\section{References}

[1] M. A. Cohen and S. Grossberg, "Absolute stability of global pattern formation and parallel memory storage by competitive neural networks," IEEE Transactions on Systems, Man, and Cybernetics, vol. 13, no. 5, pp. 815-826, 1983.

[2] H. Ye, A. N. Michel, and K. Wang, "Qualitative analysis of Cohen-Grossberg neural networks with multiple delays," Physical Review E, vol. 51, no. 3, pp. 2611-2618, 1995.

[3] L. Wang and X. Zou, "Exponential stability of Cohen-Grossberg neural networks," Neural Networks, vol. 15, no. 3, pp. 415-422, 2002.

[4] J. Cao and J. Liang, "Boundedness and stability for Cohen-Grossberg neural network with timevarying delays," Journal of Mathematical Analysis and Applications, vol. 296, no. 2, pp. 665-685, 2004.

[5] J. Park, "Robust stability of bidirectional associative memory neural networks with time delays," Physics Letters A, vol. 349, no. 6, pp. 494-499, 2006.

[6] W. Lu and T. Chen, " $R_{+}^{n}$-global stability of a Cohen-Grossberg neural network system with nonnegative equilibria," Neural Networks, vol. 20, no. 6, pp. 714-722, 2007.

[7] C. Huang and L. Huang, "Dynamics of a class of Cohen-Grossberg neural networks with timevarying delays," Nonlinear Analysis: Real World Applications, vol. 8, no. 1, pp. 40-52, 2007.

[8] Q. Liu and J. Wang, "A one-layer recurrent neural network with a discontinuous hard-limiting activation function for quadratic programming," IEEE Transactions on Neural Networks, vol. 19, no. 4, pp. 558-570, 2008.

[9] J. Cao, G. Feng, and Y. Wang, "Multistability and multiperiodicity of delayed Cohen-Grossberg neural networks with a general class of activation functions," Physica D, vol. 237, no. 13, pp. 1734-1749, 2008.

[10] Q. Zhu and J. Cao, "Stochastic stability of neural networks with both Markovian jump parameters and continuously distributed delays," Discrete Dynamics in Nature and Society, vol. 2009, no. 13, Article ID 490515, 20 pages, 2009.

[11] J. Cao and L. Li, "Cluster synchronization in an array of hybrid coupled neural networks with delay," Neural Networks, vol. 22, no. 4, pp. 335-342, 2009.

[12] J. H. Park and O. M. Kwon, "Further results on state estimation for neural networks of neutral-type with time-varying delay," Applied Mathematics and Computation, vol. 208, no. 1, pp. 69-75, 2009. 
[13] J. Park and O. M. Kwon, "Delay-dependent stability criterion for bidirectional associative memory neural networks with interval time-varying delays," Modern Physics Letters B, vol. 23, no. 1, pp. 35-46, 2009.

[14] S. Arik, "Stability analysis of delayed neural networks," IEEE Transactions on Circuits and Systems I, vol. 47 , no. 4 , pp. 568-571, 2000.

[15] J. Cao, "A set of stability criteria for delayed cellular neural networks," IEEE Transactions on Circuits and Systems I, vol. 48, no. 4, pp. 494-498, 2001.

[16] J. Cao and L. Wang, "Exponential stability and periodic oscillatory solution in BAM networks with delays," IEEE Transactions on Neural Networks, vol. 13, no. 2, pp. 457-463, 2002.

[17] J. Cao, K. Yuan, and H. Li, "Global asymptotical stability of generalized recurrent neural networks with multiple discrete delays and distributed delays," IEEE Transactions on Neural Networks, vol. 17, no. 6, pp. 1646-1651, 2006.

[18] J. Cao and Q. Song, "Stability in Cohen-Grossberg-type bidirectional associative memory neural networks with time-varying delays," Nonlinearity, vol. 19, no. 7, pp. 1601-1617, 2006.

[19] Z. Yang, D. Xu, and L. Xiang, "Exponential p-stability of impulsive stochastic differential equations with delays," Physics Letters A, vol. 359, no. 2, pp. 129-137, 2006.

[20] X. X. Liao and X. Mao, "Exponential stability and instability of stochastic neural networks," Stochastic Analysis and Applications, vol. 14, no. 2, pp. 165-185, 1996.

[21] X. X. Liao and X. Mao, "Stability of stochastic neural networks," Neural, Parallel $\mathcal{E}$ Scientific Computations, vol. 4, no. 2, pp. 205-224, 1996.

[22] S. Blythe, X. Mao, and X. Liao, "Stability of stochastic delay neural networks," Journal of the Franklin Institute, vol. 338, no. 4, pp. 481-495, 2001.

[23] L. Wan and J. Sun, "Mean square exponential stability of stochastic delayed Hopfield neural networks," Physics Letters A, vol. 343, no. 4, pp. 306-318, 2005.

[24] X. Li and J. Cao, "Exponential stability of stochastic Cohen-Grossberg neural networks with timevarying delays," in Proceedings of the 2nd International Symposium on Neural Networks (ISNN '05), vol. 3496 of Lecture Notes in Computer Science, pp. 162-167, 2005.

[25] H. Zhao and N. Ding, "Dynamic analysis of stochastic Cohen-Grossberg neural networks with time delays," Applied Mathematics and Computation, vol. 183, no. 1, pp. 464-470, 2006.

[26] J. Hu, S. Zhong, and L. Liang, "Exponential stability analysis of stochastic delayed cellular neural network," Chaos, Solitons \& Fractals, vol. 27, no. 4, pp. 1006-1010, 2006.

[27] W. Zhu and J. Hu, "Stability analysis of stochastic delayed cellular neural networks by LMI approach," Chaos, Solitons E Fractals, vol. 29, no. 1, pp. 171-174, 2006.

[28] E. Zhu, H. Zhang, Y. Wang, J. Zou, Z. Yu, and Z. Hou, " $p$ th moment exponential stability of stochastic Cohen-Grossberg neural networks with time-varying delays," Neural Processing Letters, vol. 26, no. 3, pp. 191-200, 2007.

[29] Y. Sun and J. Cao, " $p$ th moment exponential stability of stochastic recurrent neural networks with time-varying delays," Nonlinear Analysis: Real World Applications, vol. 8, no. 4, pp. 1171-1185, 2007.

[30] J. Park and O. Kwon, "Synchronization of neural networks of neutral type with stochastic perturbation," Modern Physics Letters B, vol. 23, no. 14, pp. 1743-1751, 2009.

[31] Q. Song, J. Cao, and Z. Zhao, "Periodic solutions and its exponential stability of reaction-diffusion recurrent neural networks with continuously distributed delays," Nonlinear Analysis: Real World Applications, vol. 7, no. 1, pp. 65-80, 2006.

[32] J. Liang and J. Cao, "Global exponential stability of reaction-diffusion recurrent neural networks with time-varying delays," Physics Letters A, vol. 314, no. 5, pp. 434-442, 2003.

[33] H. Zhao and K. Wang, "Dynamical behaviors of Cohen-Grossberg neural networks with delays and reaction-diffusion terms," Neurocomputing, vol. 70, no. 1, pp. 536-543, 2006.

[34] L. Wang and Y. Gao, "Global exponential robust stability of reaction-diffusion interval neural networks with time-varying delays," Physics Letters A, vol. 350, no. 5-6, pp. 342-348, 2006.

[35] Z. Yang and D. Xu, "Global dynamics for non-autonomous reaction-diffusion neural networks with time-varying delays," Theoretical Computer Science, vol. 403, no. 1, pp. 3-10, 2008.

[36] H. Zhao and Z. Mao, "Boundedness and stability of nonautonomous cellular neural networks with reaction-diffusion terms," Mathematics and Computers in Simulation, vol. 79, no. 5, pp. 1603-1617, 2009.

[37] H. Tian, "The exponential asymptotic stability of singularly perturbed delay differential equations with a bounded lag," Journal of Mathematical Analysis and Applications, vol. 270, no. 1, pp. 143-149, 2002. 
[38] A. Friedman, Stochastic Differential Equations and Applications. Vol. 1, vol. 2 of Probability and Mathematical Statistics, Academic Press, New York, NY, USA, 1975.

[39] X. Mao, Stochastic Differential Equations and Their Applications, Horwood Publishing Series in Mathematics \& Applications, Horwood, Chichester, UK, 1997.

[40] X. Li and J. Cao, "Delay-independent exponential stability of stochastic Cohen-Grossberg neural networks with time-varying delays and reaction-diffusion terms," Nonlinear Dynamics, vol. 50, no. 1-2, pp. 363-371, 2007. 


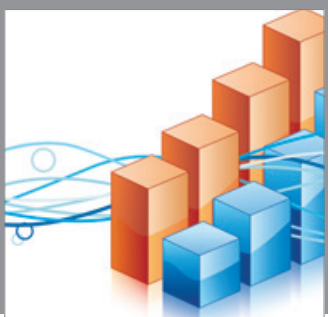

Advances in

Operations Research

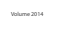

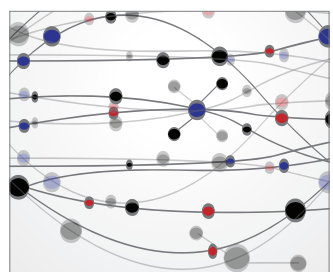

\section{The Scientific} World Journal
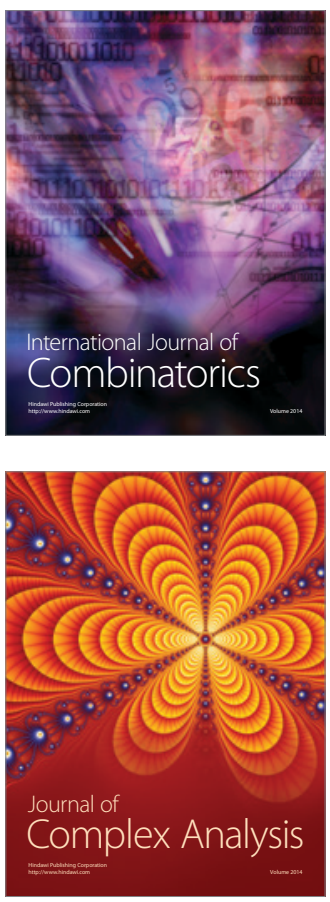

International Journal of

Mathematics and

Mathematical

Sciences
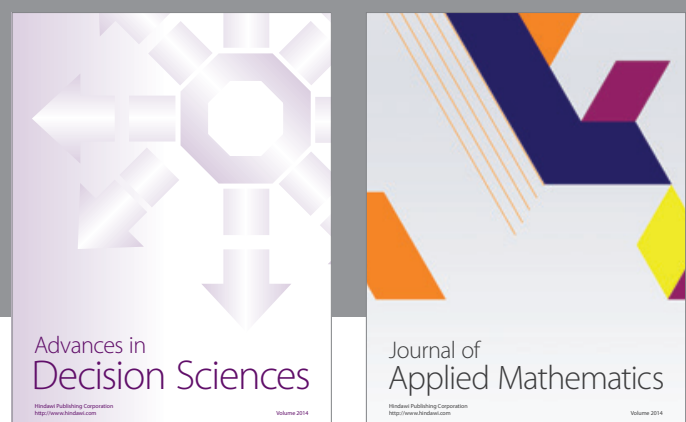

Journal of

Applied Mathematics
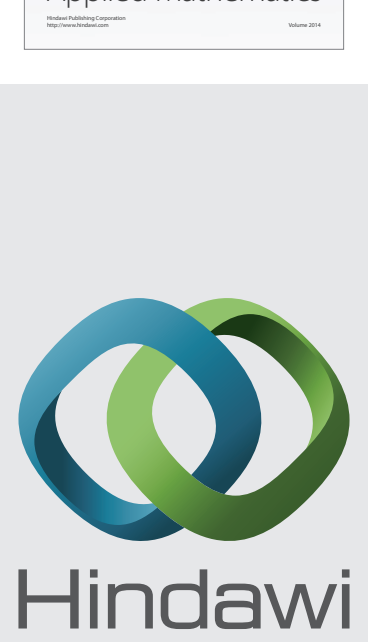

Submit your manuscripts at http://www.hindawi.com
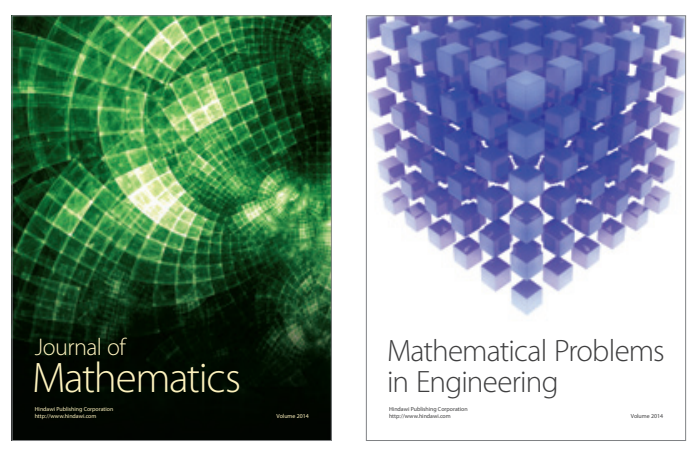

Mathematical Problems in Engineering
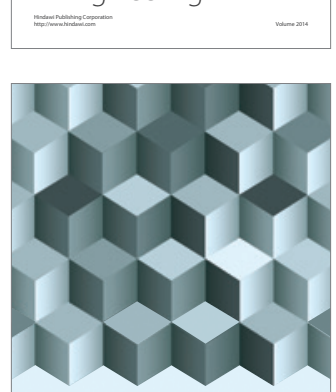

Journal of

Function Spaces
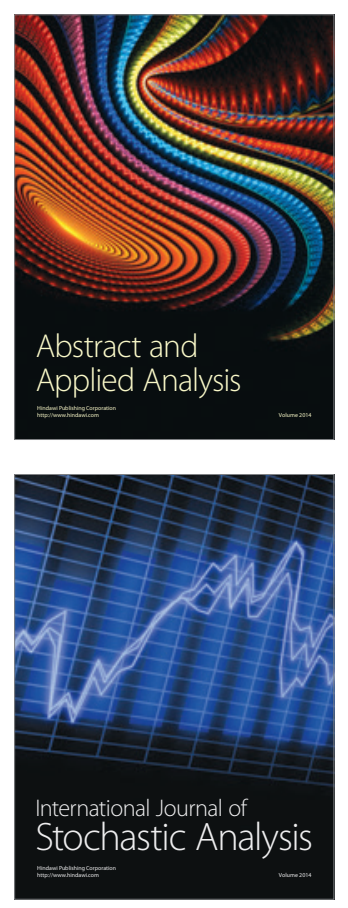

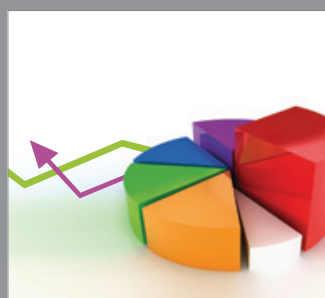

ournal of

Probability and Statistics

Promensencen
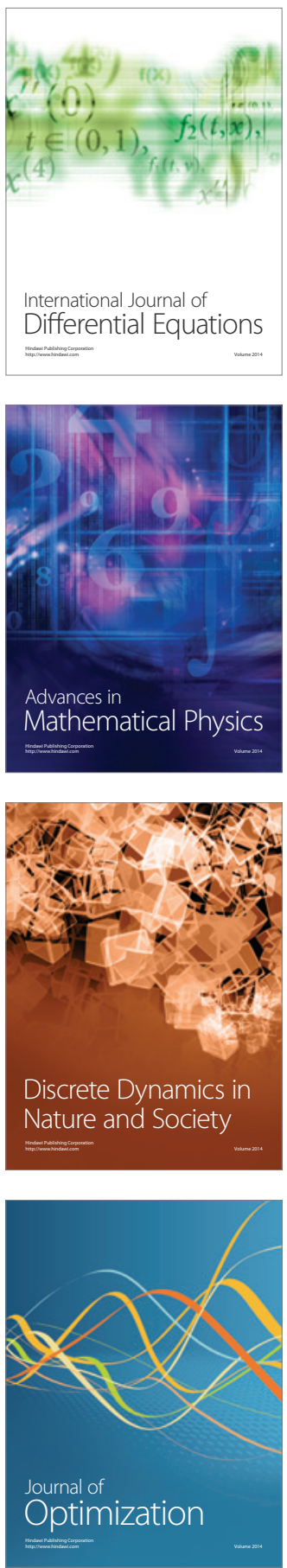